\title{
Optimal search strategies for identifying sound clinical prediction studies in EMBASE Jennifer L Holland ${ }^{1}$, Nancy L Wilczynski ${ }^{1}$, R Brian Haynes*1,2 and The Hedges Team ${ }^{1}$
}

\begin{abstract}
Address: ${ }^{1}$ Health Information Research Unit, Department of Clinical Epidemiology and Biostatistics, Faculty of Health Sciences, McMaster University, Hamilton, Ontario, L8N 3 Z5 Canada and 2Department of Medicine, Faculty of Health Sciences, McMaster University, Hamilton, Ontario, L8N 3 Z5 Canada

Email: Jennifer L Holland - hollj@mcmaster.ca; Nancy L Wilczynski - wilczyn@mcmaster.ca; R Brian Haynes* - bhaynes@mcmaster.ca; The Hedges Team - bhaynes@mcmaster.ca

* Corresponding author
\end{abstract}

Published: 29 April 2005

BMC Medical Informatics and Decision Making 2005, 5:II doi:10.1 I86/1472-6947-5-II

This article is available from: http://www.biomedcentral.com/1472-6947/5/I I

(C) 2005 Holland et al; licensee BioMed Central Ltd.

This is an Open Access article distributed under the terms of the Creative Commons Attribution License (http://creativecommons.org/licenses/by/2.0), which permits unrestricted use, distribution, and reproduction in any medium, provided the original work is properly cited.
Received: 05 January 2005

Accepted: 29 April 2005

\begin{abstract}
Background: Clinical prediction guides assist clinicians by pointing to specific elements of the patient's clinical presentation that should be considered when forming a diagnosis, prognosis or judgment regarding treatment outcome. The numbers of validated clinical prediction guides are growing in the medical literature, but their retrieval from large biomedical databases remains problematic and this presents a barrier to their uptake in medical practice. We undertook the systematic development of search strategies ("hedges") for retrieval of empirically tested clinical prediction guides from EMBASE.

Methods: An analytic survey was conducted, testing the retrieval performance of search strategies run in EMBASE against the gold standard of hand searching, using a sample of all 27,769 articles identified in 55 journals for the 2000 publishing year. All articles were categorized as original studies, review articles, general papers, or case reports. The original and review articles were then tagged as 'pass' or 'fail' for methodologic rigor in the areas of clinical prediction guides and other clinical topics. Search terms that depicted clinical prediction guides were selected from a pool of index terms and text words gathered in house and through request to clinicians, librarians and professional searchers. A total of 36,232 search strategies composed of single and multiple term phrases were trialed for retrieval of clinical prediction studies. The sensitivity, specificity, precision, and accuracy of search strategies were calculated to identify which were the best.
\end{abstract}

Results: 163 clinical prediction studies were identified, of which $69(42.3 \%)$ passed criteria for scientific merit. A 3-term strategy optimized sensitivity at $91.3 \%$ and specificity at $90.2 \%$. Higher sensitivity $(97.1 \%)$ was reached with a different 3-term strategy, but with a $16 \%$ drop in specificity. The best measure of specificity $(98.8 \%)$ was found in a 2-term strategy, but with a considerable fall in sensitivity to $60.9 \%$. All single term strategies performed less well than 2- and 3-term strategies.

Conclusion: The retrieval of sound clinical prediction studies from EMBASE is supported by several search strategies. 


\section{Background}

Clinical prediction guides (CPGs), also known as clinical prediction rules or clinical decision rules, are increasingly sought by frontline clinicians to assist in their decision making process. They provide an objective standard by which to gauge which elements in a patient's history, physical examination and laboratory tests are the most important in forming an accurate clinical assessment [1]. CPGs are created by way of deriving the rule, testing or validating the rule, and assessing the impact of the rule on clinical behaviour (impact analysis) [2-5]. CPGs vary in complexity, but those that require simple calculations on the part of the user are most recommended by CPG advocates $[5,6]$.

CPGs can serve as decision aids for determination of causation, diagnosis, prognosis, or patient responsiveness to treatment [1-3]. Some CPGs have been tailored for online or personal digital assistant (PDA) tools to aid in bedside decision-making [6]. Currently available CPGs cover a wide range of topics. For example, guides help to establish the pretest probability of pulmonary embolus [7], to determine the treatment for pharyngitis [8] and to rule out the need for unnecessary radiography for knee injuries ("Ottawa Knee Rule") [9]. CPG advocates state that, when rigorously created and appropriately applied, CPGs have the potential to influence clinical opinion, change clinical behaviour and increase efficiency while preserving or improving quality patient care and satisfaction [5].

Retrieving CPG studies from the medical literature is problematic for several reasons. First they are relatively few in quantity in comparison to other types of studies and reports posted in major, online, clinical literature databases. For example, EMBASE contains more than 9 million records and is up dated by $6000-8000$ records per week, spread over more than 4600 journal titles [10]. Second, only a fraction of CPG studies are of high quality $[1,3-5,11]$. A third problem interfering with retrieval is the plurality in terminology associated with CPG studies including test, rule, index, equation, scale, score, profile, prognosis, risk estimate, and model. Fourth, and coupled with varied terminology, is the lack of standardized controlled indexing vocabulary assigned to CPG studies, which precludes their easy extraction from large databases. Finally, studies show that clinicians lack searching skills and have little time to devote to the task of finding high quality studies on which to base their clinical practice $[12,13]$.

Several research groups have identified search strategies ("hedges", in library parlance) for MEDLINE for topics such as etiology [14], diagnosis [15], prognosis [16,17], treatment [18] and review articles [19]. Our own studies of MEDLINE retrieval $[16,17]$ led to the creation of the
PUBMED Clinical Queries search tool http:// www.ncbi.nlm.nih.gov/entrez/query/static/clinical.html.

For CPG study retrieval, 2 papers have reported filters that can be applied to MEDLINE. Ingui and Rogers [2] hand searched 4 to 6 selected journals for the years 1991 through 1998 for studies that described the development, validation or evaluation of a CPG, then developed and tested several search filters. Wong et al published CPG search strategies for MEDLINE developed by comparing hand searching 161 journals published in the year 2000 with several search terms and applying comparatively, more strict methodological quality criteria [20].

Despite the widespread use of EMBASE, especially in the UK and Europe, little is reported about methodological search filters. Bachmann et al [21] and Wilczynski and Haynes [22] reported high performance search strategies for diagnosis studies in EMBASE. In another study, Watson and Richardson tested searches against EMBASE, MEDLINE and PsycInfo for finding randomized controlled trials of cognitive therapy for depression [23]. No studies have yet been published on the retrieval properties of search terms and phrases for CPG studies in EMBASE. To fill this gap in the literature, we applied similar methodology to that used for the identification of CPG study search strategies in MEDLINE [20]. In this report, we describe the information retrieval properties of single terms and combinations of terms for identifying methodologically sound studies of clinical prediction guides in EMBASE.

\section{Methods}

We compared the retrieval performance of methodologic search terms and phrases in EMBASE with a manual review of every article for each issue of 55 journal titles for the year 2000. Originally, 170 journal titles were selected based on recommendations of clinicians and librarians, Science Citation Index Impact Factors provided by the Institute for Scientific Information, and ongoing assessment of their yield of studies and reviews of scientific merit and clinical relevance for the disciplines of internal medicine, general medical practice, mental health, and general nursing practice (list of journals provided by the authors upon request). Of these 170 journals, 135 were indexed in EMBASE. In previous work on search strategy development in MEDLINE, we determined that estimation of search term performance was not substantively affected by using smaller journal subsets, focusing on journals publishing at least some methodologically rigorous articles [unpublished data], and these smaller subsets greatly simplify data processing. Hence, 135 journals were further reduced to a 55 journals that were found to contain at least one study that met our criteria for scientific merit. 
When previously developing search strategies for some categories of articles (e.g., therapy, prognosis) for MEDLINE, we split the database into $60 \%$ and $40 \%$ components to provide a development and validation database. We subsequently found that the comparison between development and validation database results was not statistically significant [24]. For CPG search strategy development for EMBASE, it was not feasible to split the database, as there were too few "pass" articles (e.g., 69 pass CPG articles in EMBASE). Thus, search strategies were developed using the entire database.

Six research staff were rigorously calibrated for hand searching before reviewing the 2000 literature and interrater agreement for application of all criteria exceeded $80 \%$ beyond chance [25]. Hand searching was performed across 55 journals titles for the year 2000, and methodologic criteria were applied to each item in each issue to determine if the article was methodologically sound for 7 purpose categories (two other types of articles, cost and qualitative studies, were also classified but had no rigor criteria). All purpose category definitions and corresponding methodologic rigor were outlined in a previous paper [25]. Clinical prediction studies were defined as having content that pertains directly to the prediction of some aspect of a disease or condition, and the following methodologic criteria were applied: 1) the guide is generated in one or more sets of real patients (training set); and 2) the guide is validated in another set of real patients (test set).

An initial list of index terms and textwords relating to studies of different purposes (clinical prediction guides, treatment, causation, diagnosis, prognosis, economics, reviews, costs, and studies of a qualitative nature) was compiled in house. The list grew with the addition of terms or phrases suggested by clinicians, librarians and known searchers in the United States and Canada, made upon our request. From here, we compiled a list of 5385 searching terms, of which 4843 were unique and 3524 returned results (terms available on request) for retrieval of studies across all of the purpose categories. Among the 3524 terms were 641 terms that depicted clinical prediction studies such as 'clinical prediction rule', 'derivation set', 'guide', and 'validation cohort', all as textwords; 'validation process', the index term, and the index term 'model', exploded.

The search strategies were treated as "diagnostic tests" for sound studies and the manual review of the literature was treated as the "gold standard." All CPG study search terms and phrases were run in EMBASE and an automated process determined their sensitivity, specificity, precision, and accuracy. Sensitivity for a given topic is defined as the proportion of high quality articles for that topic that are retrieved; specificity is the proportion of low quality arti- cles not retrieved; precision is the proportion of retrieved articles that are of high quality; and accuracy is the proportion of all articles that are correctly classified.

The aim of testing was to identify the best single term, 2term and multiple-term (greater than two terms) strategies that would optimize sensitivity or specificity or both sensitivity and specificity together. All combinations of terms used the Boolean OR, for example, "predict.tw. OR guide.tw.". (The Boolean AND was not used because this strategy invariably compromised sensitivity.) Next, we tested all 2-term search strategies with sensitivity at least $75 \%$ and specificity at least $50 \%$ to find multiple term strategies that were optimized for sensitivity. For optimizing accuracy in a multiple term strategy, all 2-term search strategies with accuracy $>75 \%$ were tested. In total, 36,232 search strategies were tested in the development of clinical prediction guide hedges, which represents the second largest number of strategies (next to cost effectiveness studies) tested among EMBASE strategies investigated within our group.

A logistic regression approach to developing search strategies for MEDLINE did not improve performance [26], so it was not performed for this study.

\section{Results}

Indexing information was downloaded from EMBASE for 27,769 articles of various purpose categories, identified from hand-searching the 55 journals. Of these, 163 $(0.58 \%)$ were classified as clinical prediction guides, of which 69 (42.3\%) were methodologically sound.

Table 1 shows the best single term for high-sensitivity, high-specificity, and best balance of sensitivity and specificity. The single term, predict:.tw., produced both the best sensitivity $(78.3 \%)$ and best balance of sensitivity $(78.3 \%)$ and specificity (91.6\%). High specificity (98.7\%) was achieved using the single term, validat:tw., but with a concomitant drop in sensitivity to $60.9 \%$. Precision ranged from $2.3 \%$ to $10.7 \%$ for these strategies, reflecting the low prevalence of high quality CPG studies combined with less than perfect specificity.

Combination of terms with the best results for sensitivity, specificity and optimization of sensitivity and specificity are shown in Table 2 . When multiple terms are run, nearly all measures for sensitivity and specificity improve over results for single term strategies. The 3-term strategy predict:.tw. OR exp methodology OR validat:.tw achieved the best sensitivity $(97.1 \%)$, with a specificity of $74.2 \%$. Specificity peaked at $98.8 \%$, as did precision $(10.8 \%)$ and accuracy $(98.7 \%)$, with the 2-term search phrase validation.tw. OR prediction.tw., but with a significant drop in sensitivity to $60.9 \%$. The strategy that performed best for 
Table I: Single Term with the Best Sensitivity (keeping Specificity $\geq \mathbf{5 0 \%}$ ), Best Specificity (keeping Sensitivity $\geq \mathbf{5 0} \%$ ), and Best Optimization of Sensitivity and Specificity (based on the lowest possible absolute difference between sensitivity and specificity) for Detecting Studies of Clinical Prediction Guides in EMBASE in 2000. Values are percentages (95\% confidence intervals).

\begin{tabular}{lcccc}
\hline $\begin{array}{l}\text { Search term OVID } \\
\text { search* }\end{array}$ & Sensitivity $(\mathbf{n}=69)$ & Specificity $(\mathbf{n}=\mathbf{2 7 7 0 0})$ & Precision† & Accuracy $(\mathbf{n}=\mathbf{2 7 7 6 9 )}$ \\
\hline $\begin{array}{l}\text { Best sensitivity } \\
\text { predict:.tw. }\end{array}$ & $78.3(68.5$ to 88.0$)$ & $91.6(91.2$ to 91.9$)$ & $2.3(1.7$ to 2.9$)$ & $91.5(91.2$ to 91.7$)$ \\
$\begin{array}{l}\text { Best specificity } \\
\text { validat:.tw. }\end{array}$ & $60.9(49.4$ to 72.4$)$ & $98.7(98.6$ to 98.9$)$ & $10.7(7.6$ to 13.7$)$ & $98.6(98.5$ to 98.8$)$ \\
$\begin{array}{l}\text { Best Optimization of } \\
\text { Sensitivity \& Specificity } \\
\text { predict:.tw. }\end{array}$ & $78.3(68.5$ to 88.0$)$ & $91.6(91.2$ to 91.9$)$ & $2.3(1.7$ to 2.9$)$ & $91.5(91.2$ to 91.7$)$ \\
\hline
\end{tabular}

*The search strategy is reported using Ovid's search engine syntax for EMBASE.

†Denominator varies by row. : = truncation; $\mathrm{tw}=$ textword (word or phrase appears in title or abstract).

Table 2: Combination of Terms with the Best Sensitivity (keeping Specificity $\geq \mathbf{5 0} \%$ ), Best Specificity (keeping Sensitivity $\geq \mathbf{5 0} \%$ ), and Best Optimization of Sensitivity and Specificity (based on abs [sensitivity-specificity] $<1 \%$ ) for Detecting Studies of Clinical Prediction Guides in EMBASE in 2000 . Values are percentages (95\% confidence intervals).

\begin{tabular}{|c|c|c|c|c|}
\hline $\begin{array}{l}\text { Search Strategy OVID } \\
\text { search* }\end{array}$ & Sensitivity $(n=69)$ & Specificity $(n=27700)$ & Precision $\dagger$ & Accuracy $(n=27769)$ \\
\hline $\begin{array}{l}\text { Best Sensitivity } \\
\text { predict:.tw. OR exp } \\
\text { methodology OR } \\
\text { validat:.tw. }\end{array}$ & 97.1 (93.1 to 100.0) & 74.2 (73.7 to 74.7$)$ & 0.9 (0.7 to I. 2$)$ & 74.2 (73.7 to 74.7$)$ \\
\hline $\begin{array}{l}\text { Best Specificity } \\
\text { validation.tw. OR } \\
\text { prediction.tw. }\end{array}$ & 60.9 (49.4 to 72.4$)$ & 98.8 (98.6 to 98.9$)$ & $10.8(7.7$ to 13.9) & 98.7 (98.5 to 98.8$)$ \\
\hline $\begin{array}{l}\text { Best Optimization of } \\
\text { Sensitivity \& Specificity } \\
\text { validat:.mp. OR index.tw. } \\
\text { OR model.tw. }\end{array}$ & 91.3 (84.7 to 98.0$)$ & 90.2 (89.9 to 90.6$)$ & 2.3 (1.7 to 2.8$)$ & 90.2 (89.9 to 90.6$)$ \\
\hline
\end{tabular}

*Search strategies are reported using Ovid's search engine syntax for EMBASE.

†Denominator varies by row. : = truncation; $\mathrm{tw}=$ textword (word or phrase appears in title or abstract); exp = exploded subject heading; $\mathrm{mp}=$ multiple posting - term appears in title, abstract, or subject heading.

optimization of sensitivity and specificity was validat:.mp OR index.tw. OR model.tw, measured at $91.3 \%$ and $90.2 \%$, respectively. This 3-term strategy outperformed the best single term search strategy by $13 \%$ in sensitivity, while maintaining a comparable value for specificity.

\section{Discussion}

In this study we report search filters found to be effective for the retrieval of clinical predication guide studies from EMBASE. These filters are optimized for sensitivity, specificity or best sensitivity and specificity combined, each lending the searcher unique results that can be geared to his/her needs. The strategy optimized for sensitivity should be applied in cases where retrieval of all relevant articles is key, and substantial weeding of irrelevant content is seen to be acceptable. The most specific search filter is effective when the aim of the search is to retrieve only highly relevant articles, where inclusion of all pertinent matter is less important. Where the intention is to uncover a balance of targeted hits with off topic material then the strategy that maximizes both sensitivity and specificity would be best.

When comparing the results of this study to that reported by Wong et al [20] for CPGs in MEDLINE, several similarities can be drawn. Both studies report low precision for most search strategies. Like the MEDLINE search strategies, low precision is attributed to the varying content of the EMBASE database, a small proportion of which are studies of clinical prediction guides. Precision may be improved with the application of the "AND" / "AND NOT" Boolean operators or the addition of clinical con- 
tent terms or journal subsets using the Boolean AND along with the methodology search filters, but these tactics are likely to compromise sensitivity.

Other parallels occur between the 2 reports with respect to best identified search strategies. For single terms, predict (as a textword in EMBASE, predict:.tw., and as a "multiple posting" term in MEDLINE, predict:.mp.) achieved both highest sensitivity and best optimization for sensitivity and specificity in both EMBASE and MEDLINE. Similarly, validat:.tw. generated best results for specificity in both databases.

It is interesting to note that no indexing terms contributed to the optimized search strategies for CPG studies in EMBASE or MEDLINE; textwords were the composite for all winning strategies. This finding is consistent with indexing terminology not keeping pace with research methods, and suggests a means for improving indexing and retrieval in the future.

For practical purposes, we restricted our methods filter to just 2 criteria, the use of both a training and test set. Additional criteria that could have been applied include prospective validation, stating the mathematical technique used to develop the rule, clear definition and blinded assessment of predictor variables and outcomes, and prospectively testing the effect of the rule in clinical practice [1]. Further research would be needed to determine the performance of our search strategies for these additional criteria. However, it is predictable that adding more criteria would diminish the yield of our search strategies. For example, Laupacis et al [1] found that only 3\% of studies of CPGs prospectively tested clinical use, whereas we found that $42 \%$ of CPG articles passed our filter. Thus, rather than incorporating additional criteria into the derivation of search strategies, we would suggest that the additional criteria be applied by end-users, to articles retrieved by our strategies, if relevant to their purposes.

For clinicians to be able to make optimal use of clinical prediction guides in their practice, their accessibility needs to be improved upon. This study highlights search terms that maximize the retrieval of CPG studies, as well as illustrating that there is room for improvement, especially in precision. The application of "AND" and "AND/NOT" combinations or multivariate statistical techniques may help, but this remains to be determined.

\section{Conclusion}

The retrieval of higher quality clinical prediction guides from EMBASE is facilitated by the application of search filters that have been optimized for sensitivity or specificity or both.

\section{List of Abbreviations}

Clinical Prediction Guide: CPG

\section{Competing Interests}

The author(s) declare that they have no competing interests.

\section{Authors' Contributions}

NLW and RBH prepared grant submissions in relation to this project. JH, NLW and RBH drafted, commented on and approved the final manuscript. NLW and RBH supplied intellectual content to the collection and analysis of the data. NLW participated in the data collection and all authors were involved in data analysis.

\section{Acknowledgements}

This research was funded by the National Library of Medicine, USA. The Hedges Team includes Angela Eady, Brian Haynes, Susan Marks, Ann McKibbon, Doug Morgan, Cindy Walker-Dilks, Stephen Walter, Stephen

Werre, Nancy Wilczynski, and Sharon Wong.

\section{References}

I. Laupacis A, Sekar N, Stiell IG: Clinical prediction rules. A review and suggested modifications of methodological standards. JAMA 1997, 277:488-494.

2. Ingui BJ, Rogers MA: Searching for clinical prediction rules in MEDLINE. J Am Med Inform Assoc 200I, 8:39I-397.

3. McGinn TG, Guyatt GH, Wyer PC, Naylor CD, Stiell IG, Richardson WS: Users' guides to the medical literature: XXII: how to use articles about clinical decision rules. Evidence-Based Medicine Working Group. JAMA 2000, 284:79-84.

4. Randolph AG, Guyatt GH, Calvin JE, Doig G, Richardson WS: Understanding articles describing clinical prediction tools. Evidence Based Medicine in Critical Care Group. Crit Care Med 1998, 26:1603-1612.

5. Steiner JF: Clinical prediction rules as guides to treatment. Hosp Pract (Off Ed) 1999, 34:17-20.

6. McGinn T: Practice corner: using clinical prediction rules. ACP J Club 2002, I37:AII-AI2.

7. Wells PS, Ginsberg JS, Anderson DR, Kearon C, Gent M, Turpie AG, Bormanis J, Weitz J, Chamberlain M, Bowie D, Barnes D, Hirsh J: Use of a clinical model for safe management of patients with suspected pulmonary embolism. Ann Intern Med 1998, 1 29:997-1005.

8. Walsh BT, Bookheim WW, Johnson RC, Tompkins RK: Recognition of streptococcal pharyngitis in adults. Arch Intern Med 1975, 135:|493-|497.

9. Emparanza JI, Aginaga JR, Estudio Multicentro en Urgencias de Osakidetza: Reglas de Ottawa (EMUORO) Group: Validation of the Ottawa Knee Rules. Ann Emerg Med 200I, 38:364-8.

10. STN Database Summary Sheet: EMBASE [http://www.cas.org/ ONLINE/DBSS/embasess.html]

II. Wasson JH, Sox HC, Neff RK, Goldman L: Clinical prediction rules. Applications and methodological standards. $N$ Engl J Med 1985, 3 I 3:793-799.

12. Ely JW, Osheroff JA, Ebell MH, Chambliss ML, Vinson DC, Stevermer Jj, Pifer EA: Obstacles to answering doctors' questions about patient care with evidence: qualitative study. BMJ 2002, 324:710.

13. McAlister FA, Graham I, Karr GW, Laupacis A: Evidence-based medicine and the practicing clinician. J Gen Intern Med 1999, 14:236-242.

14. Wilczynski NL, Haynes RB: Developing optimal search strategies for detecting clinically sound causation studies in MEDLINE. AMIA Annu Symp Proc 2003:719-723.

15. Bachmann LM, Coray R, Estermann P, Ter Riet G: Identifying diagnostic studies in MEDLINE: reducing the number needed to read. J Am Med Inform Assoc 2002, 9:653-658. 
16. Haynes RB, Wilczynski N, McKibbon KA, Walker CJ, Sinclair JC: Developing optimal search strategies for detecting clinically sound studies in MEDLINE. J Am Med Inform Assoc 1994, I:447-458.

17. Wilczynski NL, Walker CJ, McKibbon KA, Haynes RB: Assessment of methodologic search filters in MEDLINE. Proc Annu Symp Comput Appl Med Care 1993, 17:60I-605.

18. Adams CE, Power A, Frederick K, Lefebvre C: An investigation of the adequacy of MEDLINE searches for randomized controlled trials (RCTs) of the effects of mental health care. Psychol Med 1994, 24:74I-748.

19. Jadad AR, McQuay HJ: A high-yield strategy to identify randomized controlled trials for systematic reviews. Online J Curr Clin Trials 1993, Doc No 33:3973.

20. Wong SS, Wilczynski NL, Haynes RB, Ramkissoonsingh R: Developing optimal search strategies for detecting sound clinical prediction studies in MEDLINE. AMIA Annu Symp Proc 2003:728-732

21. Bachmann LM, Estermann P, Kronenberg C, Ter Riet G: Identifying diagnostic accuracy studies in EMBASE. J Med Libr Assoc 2003, 91:34I-346.

22. Wilczynski NL, Haynes RB: Optimal search strategies for identifying diagnostic studies in EMBASE. BMC Med in press.

23. Watson RJ, Richardson PH: Identifying randomized controlled trials of cognitive therapy for depression: comparing the efficiency of Embase, Medline and PsycINFO bibliographic databases. BrJ Med Psychol 1999, 72(Pt 4):535-542.

24. Haynes RB, McKibbon KA, Wilczynski NL, Walter SD, Werre SR, for the Hedges Team: Optimal search strategies for retrieving scientifically strong studies of treatment from MEDLINE: an analytic survey. $B M J$ in press.

25. Wilczynski NL, McKibbon KA, Haynes RB: Enhancing retrieval of best evidence for health care from bibliographic databases: calibration of the hand search of the literature. Medinfo 200I, 10:390-393.

26. Haynes RB, Wilczynski NL: Optimal search strategies for retrieving scientifically strong studies of diagnosis from Medline: analytical survey. BMJ 2004, 328: 1040.

\section{Pre-publication history}

The pre-publication history for this paper can be accessed here:

http://www.biomedcentral.com/1472-6947/5/11/prepub

Publish with Biomed Central and every scientist can read your work free of charge

"BioMed Central will be the most significant development for disseminating the results of biomedical research in our lifetime. "

Sir Paul Nurse, Cancer Research UK

Your research papers will be:

- available free of charge to the entire biomedical community

- peer reviewed and published immediately upon acceptance

- cited in PubMed and archived on PubMed Central

- yours - you keep the copyright 